\title{
Sphenopalatine Ganglion Blockade: A Review and Proposed MOdification OF THE TRANSNASAL TECHNIQUE
}

\author{
Robert E. Windsor, MD, and Scott Jahnke, DO
}

The sphenopalatine ganglion block has been utilized over the last century for a wide variety of maladies. This paper provides a brief history of the use of the sphenopalatine ganglion block, a review of the sphenopalatine ganglion anatomy, and the

Over the previous century a great number of unusual symptoms, signs, and maladies have been attributed to dysfunction of the sphenopalatine ganglion (also known as Meckel's ganglion, pterygopalatine ganglion, and sphenomaxillary ganglion). It has been associated with various pain syndromes since Sluder (1) first used the term sphenopalatine neuralgia in 1909. He described unilateral facial pain associated with signs of parasympathetic hyperactivity such as mucosal congestion, rhinorrhea, and lacrimation.

The proposed treatment was a "transnasal sphenopalatine ganglion block consisting of a cotton tipped applicator dipped in $90 \%$ 'watery' solution of cocaine hydrochlorate placed on the tip of the middle turbinate for five minutes with the additional use of nitrous oxide (2). This procedure was followed by the insertion of a 4.5-inch needle through the nostril and the placement of a $2 \%$ silver nitrate solution, $0.4 \%$ gaseous formaldehyde solution, $0.5 \%$ phenol solution with a $1 \%$ iodine wash." Risks of this technique included injection into the maxillary artery, which is immediately adjacent to the ganglion. This course was repeated up to ten times with some cases having considerable pain for 4-6 weeks post procedure.

From Georgia Pain Physicians, PC, Marietta, Georgia. Address Correspondence: Robert E. Windsor, MD, Georgia Pain Physicians, PC, 2550 Windy Hill Rd, Suite 215, Marietta, Georgia 30067.

E-mail: rwindsor@aol.com

Funding: No financial support was obtained in preparation of this manuscript.

Conflict of Interest: None diagnoses which currently warrant its use. The traditional transnasal sphenopalatine ganglion block is described and our modification of the traditional technique is proposed. A case study is described in which sphenopalatine block pain control in a pa- tient with a 20-year history of poorly controlled pain from bilateral herpetic keratitis.

Keywords: Sphenopalatine ganglion block, facial pain, trigeminal neuralgia, sympathetic block, headache.
As Ruskin (2) summarized, over the last century, blockade of the sphenopalatine ganglion (SPG) was said to be an effective treatment for asthma, angina, hiccups, epilepsy, glaucoma, headaches, neck pain, vascular spasms, facial neuralgias, blindness, low back pain, sciatica, ear ache, menstrual pain, temporomandibular joint dysfunction, and hyperthyroidism (3).

More recently, Precacci et al (4) found lessening of trigger point pain following SPG blocks on patients suffering from complex regional pain syndrome. In 1982, pilot studies at Boston University School of Medicine utilizing SPG blocks with topical application of $10 \%$ cocaine solution vs a placebo of water in patients with acute low back and musculoskeletal pain found improvement in pain symptoms and mobility (5). Also in 1982, Barre (6) found the topical application of cocaine to have beneficial results as an abortive therapeutic measure in cluster headaches. Patients were taught to apply the anesthetic at home (6). In 1985, a study by Kittrelle et al (7) found that the local anesthetic effects of $4 \%$ Xylocaine had equal therapeutic benefits as $10 \%$ cocaine in aborting acute cluster headaches. In 1986, a study by Berger et al (8) found that the use of $4 \%$ topical Xylocaine was equal in benefit to $10 \%$ cocaine and better than placebo for the relief of pain in patients with chronic lower back pain.

Currently accepted indications for the SPG block are sphenopalatine neuralgia, trigeminal neuralgia, atypical facial pain, acute migraine, acute and chronic cluster headaches, herpes zoster involv- ing the ophthalmic nerve and a variety of other facial neuralgias (9-10). The mechanism by which intranasal lidocaine alleviates the pain is not fully understood, however it is believed to reverse the parasympathetic contribution to intracranial vasodilatation by blocking the sphenopalatine ganglion (11). It is not considered a first line treatment for low back pain, sciatica, arthritis, or angina despite several studies showing statistical benefit $(8,12)$.

\section{AnAtomy}

The SPG is one of four parasympathetic ganglia in the head (Fig. 1). It is located in the pterygopalatine fossa, posterior to the middle nasal turbinate under a 1$1.5 \mathrm{~mm}$ layer of connective tissue and mucous membrane and anterior to the pterygoid canal. This superficial location allows the block to be performed with topical anesthetic or by injection $(13,14)$.

The SPG is classified as a parasympathetic ganglion because only pre-ganglionic parasympathetic axons are believed to synapse within the ganglion. As a result, it contains the cell bodies of the postganglionic parasympathetic neurons. Post-ganglionic sympathetic neurons as well as somatic sensory afferent branches of the maxillary division of the trigeminal nerve also pass through the ganglion (but do not terminate), all of which may be inhibited by blockade of the SPG (15).

The sphenopalatine parasympathetic fibers originate in the superior salivatory nucleus and emerge from the brain stem as part of the nervus intermedius of the facial nerve. These fibers branch from the 


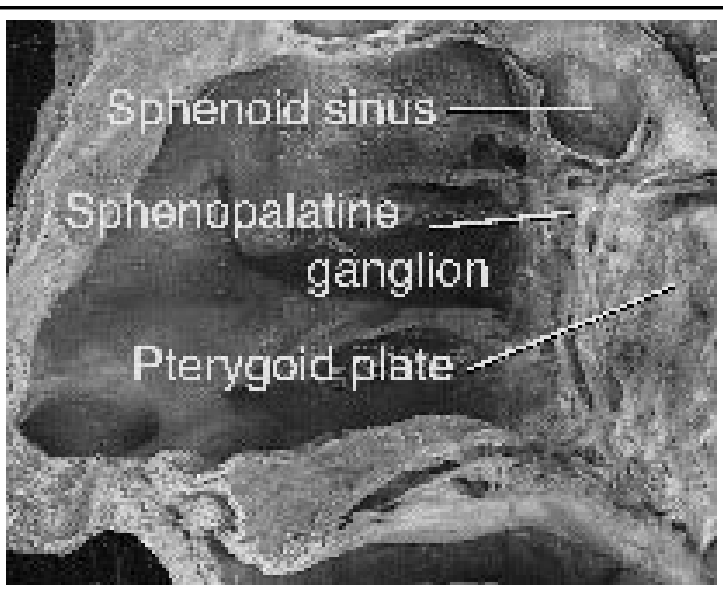

Fig. 1. Sagittal section through nasal cavity and sphenoid sinus, demonstrating the close proximity of the sphenopalatine ganglion (also known as the pterygopalatine ganglion), covered by 1 to $2 \mathrm{~mm}$ of mucosa and connective tissue, just posterior to the middle turbinate. Instilled local anesthetic can readily penetrate the mucosa and reach the ganglion.

Modified and used with permission, Rohen JW, et al. Color Atlas of Anatomy, 4th ed. Williams and Wilkins, Baltimore, 1998, pp 143.

nerve as the greater petrosal nerve, which then traverses the pterygoid canal to reach the SPG. Post-ganglionic parasympathetic neurons from this ganglion then distribute to the lacrimal gland and glands of the nasal cavity, paranasal sinuses, palate, and upper pharynx where they promote secretion (15). They are also known to innervate the major cerebral arteries in conjunction with post-ganglionic sympathetic fibers (16).

The post-ganglionic sympathetic fibers associated with the SPG synapse in the superior cervical ganglion before traveling along the internal carotid plexus and deep petrosal nerve to reach the pterygopalatine fossa where they pass through the SPG and continue on with the branches of the maxillary nerve (15).

The sensory afferent axons traveling through the SPG arise from the maxillary division of the trigeminal nerve, which enters the pterygopalatine fossa through the foramen rotundum to lie just superior to the SPG (15). The sensory fibers connect the maxillary nerve to the SPG by way of five branches that extend from the nasopharynx, nasal cavity, palate, and orbit (17). The pharyngeal branch supplies the sphenoidal sinus and the mucosa of the roof of the pharynx via the palatine canal, the greater palatine nerves extend posteriorly, and the inferior nasal branches supply the palate via the greater palatine foramen. The tonsil and soft palate are supplied via the lesser palatine nerve as it arises from the lesser palatine foramen. The nasopalatine nerve emerges through the sphenopalatine foramen, passes along the nasal septum, and emerges through the median incisive foramen to reach the hard palate. The posterior ethmoidal and sphenoidal sinuses below the periosteum of the orbit are supplied via the orbital branches. The nasal cavity is supplied via the posterior superior nasal branches (18).

\section{CASe Study}

The patient is a 42-year old female was referred to our clinic for the pharmacologic management of bilateral eye pain due to herpes keratitis. She began having eye pain about 18 years prior to the visit, but pain had increased in intensity and frequency. On a Visual Analog Scale (VAS), she rated her continual pain as a 47/10 with intermittent exacerbations rating 9-9.5/10 occurring several times per week, lasting 15-30 minutes each. She described the pain as being of a burning and shooting sensation in both eyes, cheeks, and periorbital regions. Closing her eyes reduced the pain while bright lights, windy and dry environmental conditions increased the pain.

Verification of medical records indicated that she had normal brain and orbital MRI studies, and intraocular pressures and funduscopic examinations were within normal limits. She underwent three punctual occlusion procedures in an attempt to increase eye moisture, without improvement. Multiple medications including carbamazepine, pentazocine, gabapentin, isometheptene mucate, and tricyclic antidepressants were tried without benefit. She initially was managed on hydrocodone and acetaminophen preparations and more recently, controlled release morphine with some success in controlling her symptoms. Despite these efforts she continued to have intermittent incapacitating exacerbations of the herpes keratitis.

Ultimately, the patient underwent a left transnasal SPG block. Prior to the block, the patient's VAS was 9.5/10. After the block with TAC (tetracaine, adrenalin, and $10 \%$ cocaine), she reported that the facial pain was reduced to a $3 / 10$ on the right and a 7/10 on the left. She experienced about a week of relief prior to return of pain. This procedure was repeated on the left once and her symptoms similarly abated for one month prior to return. The procedure was performed on the right side and her right sided symptoms pain completely abated for one month prior to return. Upon return, exacerbations were much less frequent even though her baseline pain returned to its normal range. Currently, she is being maintained on monthly bilateral SPG blocks with TAC and her opioid consumption has been reduced by $50 \%$.

\section{TECHNIQUE}

In our ambulatory surgery center, patient preparation for a transnasal sphenopalatine ganglion blockade consists of thorough patient education of the procedure, risks, benefits, expected outcome, written informed consent, and large bore intravenous access. Patient monitoring should include blood pressure and heart rate monitoring. If TAC (tetracaine, adrenaline, and cocaine) or cocaine solutions are utilized, then cardiac monitoring and pulse oximetry are advised to observe any potential cardiac effects.

The patient is placed supine on the table with the cervical spine extended. The anterior nares are inspected for polyps, tumors, foreign bodies, or significant septal deviation. A small amount of $2 \%$ viscous lidocaine is instilled into the nare(s) being treated. The patient is asked to briskly inhale drawing the lidocaine toward the posterior nasal pharynx. This 
lubricates and anesthetizes the mucous membranes making the procedure more comfortable.

Several authors describe the traditional technique using sterile $10 \mathrm{~cm}$ cotton tipped applicators that are dipped in the chosen anesthetic and then advanced along the superior border of the middle turbinate, until it reaches the posterior wall of the nasopharynx $(9,10,19)$. Some techniques describe dripping one or two $\mathrm{ml}$ of the anesthetic along the shaft of the applicator. The applicators are removed after 20 -30 minutes

The patient commonly will complain of a bitter taste in the mouth from the medication dripping down from the nasopharynx, a slight numbness in the back of the throat. and slight lacrimation. Occasionally, the patient may complain of slight lightheadedness that usually resolves 20-30 minutes after the procedure. The difficulty in utilization of these techniques lies in the inability to reasonably quantify the amount of anesthetic that actually comes into contact with the posterior nasopharynx.

\section{New Technique}

We would like to propose a new technique of performing the SPG block that should allow better control and quantification of the amount of medication reaching the posterior nasopharynx. Utilizing a standard intravenous administration set (B. Braun Medical CSP152VSL, Bethlehem, PA USA), the tubing is unfurled and the flow regulator is moved tight against the distal Y-infusion port

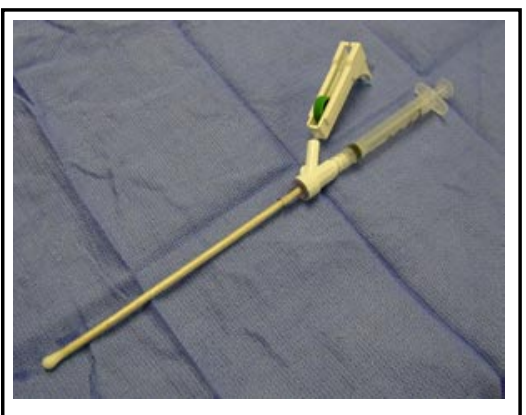

Fig 2. A picture of the suggested SPG block device. Note that the cut end of the tubing reaches the proximal end of the cotton tip of the applicator and the infusion port lies immediately against the proximal tip of the applicator. (stop cock); a $3 \mathrm{~mL}$ syringe can be attached to the port. The distal tubing is then measured against the $10 \mathrm{~cm}$ cotton tipped applicator. The length of the tubing is cut so that when the tubing is slid over the cotton tipped applicator, the cut end of the tubing reaches the proximal end of the cotton tip of the applicator and the infusion port lies immediately against the proximal tip of the applicator (Figs. 2 and 3).

At this point, the patient's nare(s) are anesthetized by injecting 1-2 $\mathrm{mL}$ of $2 \%$ viscous lidocaine with a $3 \mathrm{~mL}$ syringe without a needle. The SPG block device is then inserted through the ipsilateral nare along the middle turbinate to the nasopharynx in the case of an ipsilateral pain syndrome, or one nostril at a time with both nares ultimately being injected when the pain syndrome is bilateral. A syringe containing $2.0 \mathrm{~mL}$ of TAC solution is attached to the Y-infusion port and slowly injected through the catheter-cotton tipped applicator set up (Figs. 4 and 5). We found the dead space in the tubing and the amount of solution to adequately wet the cotton tip of the applicator to be $0.5 \mathrm{~mL}$. One half $\mathrm{mL}$ of the treatment solution should be injected immediately to fill the dead space of the catheter and soak the cotton tip of the applicator. The next $1.5 \mathrm{~mL}$ of treatment solution should be injected over the next 5-10 minutes. This procedure reliably delivers $1.5 \mathrm{~mL}$ of solution to the posterior pharynx, but has the added benefit of soaking the cotton tip of the applicator so that up to $0.5 \mathrm{~mL}$ of solution remains in contact with the nasopharynx mucosa for the duration of the procedure. The applicator(s) are left in placed for 20 to 30 minutes and

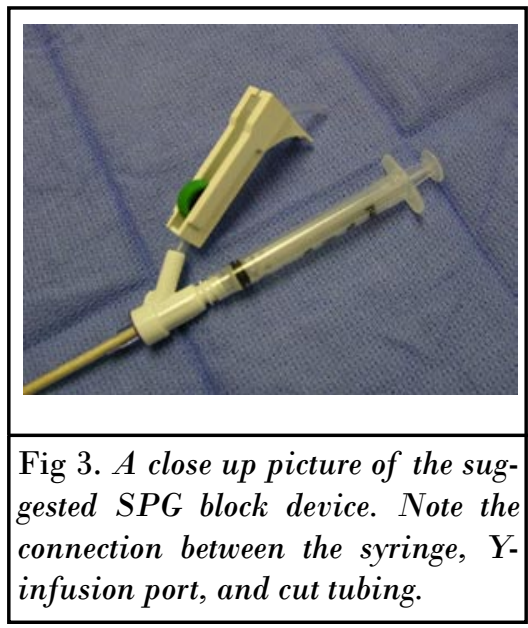

removed. The patient is the moved to the recovery area for standard post procedure monitoring.

\section{DISCUSSION}

The transnasal technique presented here has been developed to provide increased patient acceptance of the inherent discomfort of placing multiple applicators in the nares. The technique allows increased control of targeting and delivery of treatment solution reaching the posterior nasopharynx. While the application of $1-2 \mathrm{~mL}$ of $2 \%$ lidocaine to the nasal membranes typically results in minimal and manageable systemic side effects, the application of $2 \mathrm{~mL}$ of $10 \%$ cocaine may result in more profound cardiovascular side effects. By slowly injecting the treatment solution over 5-10 minutes, if the patient has changes in any of the parameters being monitored or worrisome subjective side effects (e.g., shortness of breath or chest pain), the procedure may be aborted by simply removing the applicator without injecting more medication. In previously described techniques, the medication has already been applied to the cotton tipped applicator and the treating physician may not be certain how much medication is actually applied to the nasopharynx by the time the patient exhibits altered vital signs or subjective side effects.

\section{Conclusion}

In summary, this transnasal technique for sphenopalatine ganglion block uses a single applicator in performing the block, which provides increased patient comfort, prolonged application of the medication to the tissue overlying

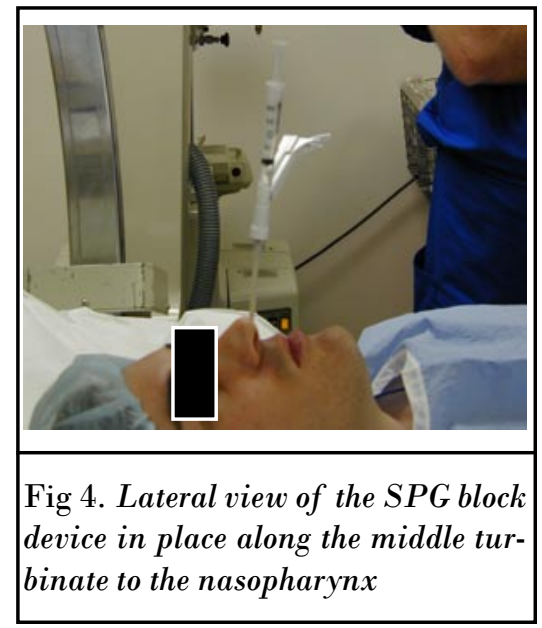




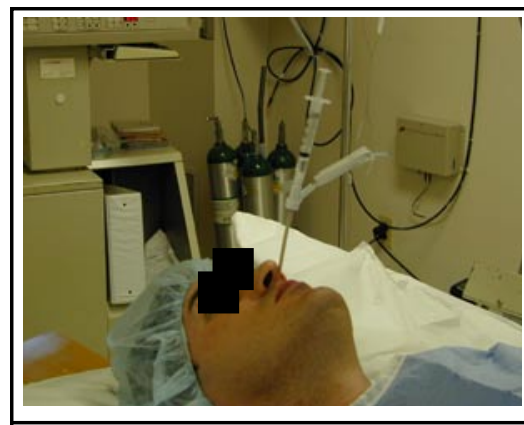

Fig 5. Oblique view of SPG block device in place along the middle turbinate to the nasopharynx.

the sphenopalatine ganglion, and a controlled, incremental infusion of local anesthetic or other treatment solution to the target. This may maximize safety and improve results.

\section{Author Affiliation:}

\section{Robert E. Windsor, MD}

President

Georgia Pain Physicians, PC

Program Director, Emory/Georgia

Pain Physicians Pain Management

Fellowship

2550 Windy Hill Rd., Suite 215

Marietta, Georgia 30067

E-mail: rwindsor@aol.com

\section{Scott Jahnke, DO}

Fellow

Emory/Georgia Pain Physicians Pain

Management Fellowship

2550 Windy Hill Rd., Suite 215

Marietta, Georgia 30067

\section{REFERENCES}

1. Sluder G. The anatomical and clinical relations of the sphenopalatine ganglion to the nose. NY State J Med 1909; 90:293298.

2. Sluder G. Nasal Neurology, Headaches and Eye Disorders. St. Louis, CV Mosby, 1927.

3. Ruskin A. Sphenopalatine (nasal) ganglion: Remote effects including "psychosomatic" symptoms, rage reaction, pain, and spasm. Arch Phys Med Rehabil 1979; 60: 353-359.

4. Procacci P, Francini F, Zoppi $M$ et al. Cutaneous pain threshold changes after sympathetic block for reflex dystrophies. Pain 1975; 1:167-175.

5. Reder M, Hymanson A, Reder M. Sphenopalatine ganglion block in treatment of acute and chronic pain. In Hendler NH, Lond DM, Wise TN (eds.) Diagnosis and Treatment of Chronic Pain. Boston, John Wright, 1982, pp 97-109.

6. Barre F. Cocaine as an abortive agent in cluster headaches. Headaches 1982; 22: 69-73.

7. Kittrelle J, Grouse D, Seybold M. Local anesthetic abortive agents. Arch Neurol 1985; 42:496-498.

8. Berger J, Pyles ST, Saga-Rumly S: Does topical anesthesia of the sphenopalatine ganglion with cocaine or lidocaine relieve low back pain. Anesth Analg 1986; 65: 700-702.

9. Raj P, Lou L, Erdine $S$ et al. Radiographic imaging for regional anesthesia and pain management. New York, Churchill Livingstone, 2003, pp 66-71.
10. Waldman S. Atlas of Interventional Pain Management. Philadelphia, WB Sanders, 1998, pp 10-12.

11. Edvinsson L. Innervation and effects of dilatory neuropeptides on cerebral vessels. Blood Vessels 1991; 28:35-45.

12. Reder M, Hymanson A, Reder M. Sphenopalatine ganglion block in treatment of acute and chronic pain. In Hendler N, Lond D, Wise T (eds). Diagnosis and Treatment of Chronic Pain. Boston, John Wright, 1982, pp 97-109.

13. Waldman S. Sphenopalatine ganglion block- 80 years later. Reg Anesth 1993; 18:274-276.

14. Salar G, Ori C, lob I. Percutaneous thermocoagulation for sphenopalatine ganglion neuralgia. Acta Neurochir (Wien) 1987; 84:24-28.

15. Waxman S: Correlative Neuroanatomy, 23rd ed. Stamford, Appleton \& Lange, 1996, pp 265-266.

16. Hardebo J, Arbab M, Suzuki N et al. Pathways of parasympathetic and sensory cerebrovascular nerves in monkeys. Stroke 1991; 22:331-342.

17. Eagle W. Sphenopalatine neuralgia. Acta Otolaryngol 1942; 35:66-84.

18. Gardner G, Gray A, O'Rahilly S: Anatomy: A Regional Study of Human Structure, 5th ed. Philadelphia, WB Sanders, 1906, pp 676-677.

19. Windsor R, Gore H, Merson M: Interventional sympathetic blockade. In Lennard T (ed.) Pain Procedures in Clinical Practice, $2^{\text {nd }} e d$. Philadelphia, Hanley \& Belfus, 2000, pp_321-324. 